\title{
Discounting Lives: Analyzing the causes of Hispanic Adversity raging in the Healthcare sector during Covid-19 using Cumulative Inequality Theory.
}

\author{
Paras Bhatt \\ The University of Texas at San \\ Antonio \\ paras.bhatt@utsa.edu
}

\author{
Yuanxiong Guo \\ The University of Texas at San \\ Antonio \\ yuanxiong.guo@utsa.edu
}

\author{
Yanmin Gong \\ The University of Texas at San \\ Antonio \\ yanmin.gong@utsa.edu
}

\begin{abstract}
In the wake of the devastation caused by Covid-19 in the community, one racial minority has been the most heavily hit by this pandemic - Hispanics. There have been numerous studies that bring out the difference in the level and quality of healthcare received by racial and ethnic minorities. However, most of these studies have focused on using socioeconomic status to account for minorities being disproportionately affected by health-related issues and ailments. We investigate the cumulative and ever-increasing gap in healthcare facilities and the resultant inequality which leads to Hispanics being severely affected by the novel coronavirus more than any other race or ethnicity. The study highlights the importance of considering a cumulative inequality effect that can help explain the reason for minorities being more prone to Covid-19. Even with an increase in their socioeconomic status, Hispanics have a way higher infection rate than other races. It is here that we use the cumulative inequality theory to explain the counterintuitive observation above. A cumulative inequality in healthcare facilities over the years helps to account for the disproportionate infection rate among the Hispanic population. We conduct empirical case comparisons (ECCS) to test our hypotheses and find that socioeconomic status is not sufficient to explain the higher infection rates among the minority population. We propose using cumulative inequality theory to fight both the current infection rate among minorities and fortify them from being negatively affected by future pandemics as well.
\end{abstract}

\section{Introduction}

Covid-19 has rampaged many of the world's countries today. It is ever more evident in no other country than USA which ranks the highest in infections and deaths. While looking from afar there appear to be giant blimps in the form of high infection rates in New York, California, and Florida. However, when taking a closer look at the victims of this virus it is clear that minorities suffer more than the Anglo population both in terms of increased infections and deaths [1, 18]. Infection rate, deaths, hospital admission, and even Medicare access, looking at these metrics we see Hispanics facing an impending health and financial burden [19]. Minority populations are at a disadvantage even in areas where healthcare facilities are better than some Anglo areas. The reason for this is the racial and income inequality that has been present in American society for quite some time and has now been thrust into the spotlight because of this pandemic.

This pandemic has evolved into a sinkhole for the Hispanic population. Although they are a minority race in the US population, but surprisingly make up a majority of the Covid-19 cases in the country [22]. Also, this is not just a one-off phenomenon, over the decades Hispanics and other minority racial and ethnic groups have always been at a disadvantage when receiving care in the healthcare sector $[2,3]$. With each passing year the inequality in the level of healthcare facilities available to minorities, especially Hispanics, has been increasing at a rapid pace [57]. The gap between the provincial haves, here the Anglo population, and the have-nots, here the racial minorities, has formed a crater that showcases the deep divide in the quality of healthcare services afforded to ethnic minorities. All of this has finally culminated to disproportionately hit the Hispanic population during this pandemic with an increased number of hospitalizations, infections, and deaths than Anglo populations [18]. The higher number of infections among the Hispanic community may seem surprising to some people from the outside, but for others, it is a stark representation of their reality playing out yet again in a time of crisis.

Research has been rife in investigating the factors responsible for this deep divide in healthcare. Socioeconomic status is one of the frequently used factors [58]. It is made up of two components - income and level of education. Many studies have used socioeconomic status to highlight the gap in the level of care received by rich vs poor or people with college 


\section{Cumulative Inequality causing Hispanic Adversity in Healthcare}

degrees vs people without degrees [4,5]. However, as stated in these works, race and socioeconomic position in US are so closely intertwined that it is difficult to isolate racial/ethnic disparities in healthcare due to socioeconomic disparities. Also, there is little evidence to suggest that white and non-white ethnicities differ in their ability to transform socioeconomic resources into good health. Further, socioeconomic status is an individual factor used for explaining racial inequality but there also exist institutional factors contributing to the divide over healthcare facilities for ethnic minorities.

We study the impact of these institutional factors by specifically looking at the healthcare gap through a confluence of cumulative inequality theory [6] and the weathering hypothesis [7]. We argue that even though socioeconomic status is a good indicator of individual inequality, there is a need to identify institutional factors that are responsible for ethnic minorities, particularly Hispanics, being disproportionately affected year after year by serious diseases [59]. We conduct empirical case comparisons of rich and poor counties in US and show that because of being at a disadvantage in the healthcare sector for many years, there now exists a cumulative inequality that affects the level and quality of healthcare services available to the Hispanic population. Our analysis shows that even when the socioeconomic marker of household income increased for Hispanic families, it still did not lead to an increase in the level of healthcare facilities accessed by them. Also in another comparison we saw that, similar to income, when the number of medical institutions in Hispanic dominant counties went up the level of healthcare facilities accessed by them did not. These counterintuitive findings support our hypothesis about the existence of institutional factors that affect racial minorities and their access to healthcare services in the community.

The research questions we seek to answer with this study are: 1) Whether individual socioeconomic characteristics, such as Household Income, are sufficient to explain the level of healthcare facilities used by racial and ethnic minorities. 2) Do institutional factors exist that can better predict and explain the usage level of healthcare facilities by ethnic minorities. 3) Has the decades-long weathering effect given rise to a cumulative inequality in the healthcare industry, because of which current racial and ethnic minorities in US with better socioeconomic resources still cannot access a higher level of healthcare facilities?

The rest of the paper is structured as follows: In section 2 we conduct a literature review of research on racial inequality and disparities in healthcare. In section 3 we explain the theoretical frameworks of the weathering hypothesis and the cumulative inequality theory. In section 4 we present our research model and explain our logic for hypotheses generation. In section 5 we discuss our methodology, outline data sources, conduct data analysis, and explain our empirical case comparisons of two representative US counties. In section 6 we discuss our findings, contributions, and implications from this research. We conclude the paper in section 7 by outlining our future efforts and conclusions.

\section{Literature Review}

From the annals of decades of research over the years, a consistent observation in the US healthcare sector has been the presence of racial differences and the existence of inequalities in the level of care afforded to racial and ethnic minorities [8]. These inequalities are consistent in the context of both physical and mental health. One of the early explanations provided by the research community was the presence of racism. Racism, as a determining factor for perpetuating inequalities in healthcare, has been investigated in several studies over the years $[9,10]$. Inferior treatment by healthcare providers, personal biases of doctors, and a lack of marketing of health insurance to ethnic minorities are some of the reasons highlighted in the studies that use racism to explain the low level of healthcare facilities use by minorities.

Many studies focused on using socioeconomic studies as a marker to determine whether racial and ethnic minorities could actually afford healthcare or not $[4,5]$. These works used two major components to make up the variable of socioeconomic status. First, the income earned by an individual is considered as being representative of them being able to access healthcare facilities. Second, the education level attained by the individuals were considered to be an identifying factor that could explain healthcare usage among minorities. Socioeconomic status has been used widely among the racial inequality domain to not only investigate unequal access to healthcare but also explain why ethnic minorities are more likely to spend a greater number of years of their lives with chronic health conditions than white only populations [11]. Less income and education are identifiers associated with racial minorities and because of these reasons a vicious cycle of inequality takes hold in ethnic communities where poorer households cannot access healthcare for want of income and even well to do households are not marketed or know about healthcare facilities because providers believe in stereotypes and view all minorities as having low socioeconomic status than the Anglo population.

In some cases, the health maintenance organizations (HMO) responsible for providing healthcare facilities may choose to neglect the groups that need it the most 


\section{Cumulative Inequality causing Hispanic Adversity in Healthcare}

and engage in reverse targeting. This means they allocate healthcare resources to those who need it the least like those living in well-off and affluent white neighborhoods rather than ethnic communities that are in dire need of such resources [12]. Such predatory practices also contribute to ethnic minorities being excluded from the healthcare sector completely and lead them to be disillusioned about the healthcare sector as a whole. An example of this is when an HMO decides to conduct immunizations for children at the lowest level of risk and overlook those kids in less affluent areas where ethnic minorities reside. However, the HMO may still maintain a good record by showing a sufficient number of immunizations being administered.

Many theories have been used in the literature to study discrimination and racial inequality. Some of them include the ecosocial theory [13], weathering framework [14], and cumulative inequality theory [15]. There have been efforts to look at social sources such as residential segregation that give rise to racial disparities in healthcare access [16]. As racial inequality is first seen in small neighborhoods and pockets of the racial communities, which then manifests itself across the society, it is important to consider how ethnic minorities are treated, what opportunities they have at their disposal, where do they live and work. All these factors are identified as some of the determining variables that can contribute to inequality in accessing healthcare facilities. Research has even looked at the lack of ethnic diversity in the healthcare workforce and consider it an important reason as to why ethnic populations refrain from using healthcare facilities [17]. Since racial inequality in the healthcare sector is also a topic of investigation under the medical knowledge domain, a cross-functional research paradigm is needed to understand the Hispanic adversity.

The Hispanic population has been negatively affected by stereotyping during the Covid-19 pandemic to a great extent, perhaps even greater than any other racial or ethnic minority in the US [18]. They are naturally spooked by the disproportionate infection rate and its implications on both their health and finances [19]. Even the US Center for Disease Control (CDC) has published a guide detailing why racial and ethnic minorities are more likely to be affected by Covid-19 [20]. The ethnic populations that are the most affected by this pandemic are Hispanics who now begin to question the status quo as to why people of their ethnicity are being more affected by it.

The answer is obscured by years of status generalizations, stereotyping, inequality, and economic disadvantage [47] which has given rise to a cumulative inequality effect. Even if Hispanics are not at an economic disadvantage, they are still likely to be negatively affected by the pandemic as evidenced by the current Covid-19 pandemic [1] Even in counties where the socioeconomic status of Hispanics is much higher than the Anglo population there, it is still the Hispanic population which shows a worse infection rate. Therefore, we see a pattern of cumulative inequality that has formed over the years because of being weathered and exposed to the same disadvantageous situations year after year. We elaborate on these hypotheses in our next section. Similarly, even if Hispanic communities now have a greater number of medical institutions within their area, they are still likely to have a higher infection rate than the non-Hispanic population. These two observations make up the crux of our weathering framework based cumulative inequality theory argument.

\section{Theoretical Background}

We use two main social paradigms to guide our theoretical framework for this paper - the Cumulative Inequality Theory [6] and the weathering hypothesis [7]. We combine them to explain how it gave rise to the perfect storm to form the Hispanic Adversity that is now very much evident in the healthcare sector during this Covid-19 pandemic.

\subsection{Weathering Framework}

Geronimus proposed the weathering hypothesis as a possible explanation for the poor health of young African American women participants in her study about infant mortality [6]. She proposed that, compared to non-Hispanic white women, the average African American woman's health begins to deteriorate at an earlier age because of the cumulative socioeconomic disadvantage faced by them in life. It is because of a persistent lack of healthcare facilities that are everpresent in neighborhoods with a large population of racial and ethnic minorities. This hypothesis brings to light the fact that, even though the medical field has undergone rapid advances in the level of healthcare facilities, these have not trickled down to racial and ethnic minorities living on the fringes of the healthcare sector.

Due to the consistently low levels of healthcare services, a weathering effect takes shape. Year after year of being faced with the same disadvantages changes how these ethnic communities look at the healthcare industry, and they grow more distrustful of the healthcare system in general [21]. The lack of healthcare services then weathers the population down and just like the African American women in Geronimus' study, the Hispanic community too has now become more prone to healthcare conditions. This is clearly self-evident when we take a closer look at the racial and ethnic data 


\section{Cumulative Inequality causing Hispanic Adversity in Healthcare}

related to the infection and death rate of Covid-19 patients today [22]. We use this hypothesis and attach it to the cumulative inequality theory because they have similar frames of reference and can be effectively sewn together to make sense of the adversity faced by the Hispanic community during this pandemic. It is not just a question of researching why, but more so of understanding how it happened.

\subsection{Cumulative Inequality Theory}

Merton's articulation of the Matthew effect [23] in science is one of the driving forces of the cumulative inequality theory and can be succinctly used to explain it in a very simple way. The Matthew effect is very similar to the idea that - the rich get richer and the poor get poorer. Using this effect, we can also form a basic understanding of the cumulative inequality theory. The central idea is as follows: Consider there are two groups - groups one and two. Now, group one receives an initial advantage, then group two is automatically at a disadvantage. Also, this advantage is cumulative so the next time, group one is bound to receive the advantage again because they received it the first time. This goes on up until a point where the gap between the two groups is so large that, even if in some scenario an advantage were to be given to group two it would fail to materialize into an actual benefit for them. Thus, this theory is primarily concerned about how cumulative advantage helps certain groups and the resulting cumulative disadvantage hinders certain other groups.

Cumulative Inequality has been studied in the healthcare domain to bring out the racial disparities in health insurance coverage faced by ethnic minorities [24]. Kail and Taylor used data from the Health and Retirement Study (1996 - 2008) to show that market insurance is an important mediator of the limitation face by African Americans. Apart from racial undertones studies have also used this theory to understand the generational gaps even within a family i.e. among grandparents, parents, and children. Gilligan et. al. [25] researched how this inequality is transmitted along family lines and affects their life course. Another study conducted by Jackson [26] looked at the effect of this inequality on children and whether the time and duration of the inequality play a role in their academic achievement.

Many of the studies have looked at individual factors, such as socioeconomic status, responsible for the consistent disadvantages faced by ethnic minorities in healthcare. However, as we have now learned from this pandemic, there is a need to consider institutional factors that contribute to perpetuating this inequality and result in adversities for ethnic minorities, especially for ones in the Hispanic community. To the best of our knowledge, our work is the first to combine the weathering framework with cumulative inequality, both of which can help to present the crater in healthcare services and facilities available to ethnic communities. We also provide evidence of the existence of an institutional factor other than socioeconomic factors such as income which is not sufficient to paint a clear picture of the adversities faced by communities of color in times of this pandemic.

\section{Research Model}

We present our hypotheses based on a weathering effect [7] which has led to cumulative inequality in the healthcare facilities [6] afforded to racial and ethnic minorities. We use current events as well as past studies to drive our hypotheses generation efforts.

There have been numerous reports highlighting how racial and ethnic minorities have been hit the hardest by Covid-19 [20]. The racial disparities are even more evident for the Hispanic community which is currently facing the brunt of the pandemic and makes up a majority of the cases of infections and deaths across US $[1,18,19,22]$.

Racial segregation with respect to contracting infectious diseases has been studied widely in the annals of medical research. Specifically, Acevedo-Garcia [37] explained a conceptual framework that showcases the increasing gap in the incidence of infectious diseases between minorities and the Anglo majority. This increasing gap points to the existence of cumulative inequality between the races, with minorities being more susceptible to infectious diseases. In a community setting when we look at immunity levels among races, there exists a similar gap. Strully [36] attributes increased exposure to flu infections to lack of vaccinations much more prevalent in minorities than Anglo populations.

The current disparity in Covid-19 infections for Hispanic population and the existence of racial factors that underline this inequality leads us to our first hypothesis:

H1 - Compared to Anglo communities, Hispanics will have a positive relationship with the Coivd-19 infection rate.

The weathering hypothesis has been researched by scholars for decades and most of the studies point to the existence of a disparity in healthcare services available to the racial and ethnic minorities because of it [7, 27]. In US, Federal investments and government initiatives are seen as essential in addressing these disparities [39]. Consequently, over the years there have been many government led efforts to address the health and economic disparities among minority population. Most of them have focused on alleviating some of the factors 
responsible for such weathering effects on racial communities. such as low socioeconomic status and lack of access to healthcare institutions [28]. Two notable initiatives that especially look at the healthcare related disparity are led by United States Department of Health and Human Services. First, HHS Action Plan to Reduce Racial and Ethnic Health Disparities and second, Office of Minority Health's Healthy People program [38]. A common point within these is the goal of increasing the number of institutions providing primary care health services to minorities. Also, US Department of Commerce offers various socioeconomic initiatives that aim to increase minority participation in the economy. Minority Business Development Agency (MBDA) is one agency that specifically works for minority populations [40]. There have been various initiatives that aim to increase diversity in the workforce, and efforts to increase education levels (through minority serving institutions) which is another socioeconomic indicator apart from income.

As a response to the evident disparities in health and socioeconomic status of racial minorities several initiatives were launched to address this inequality. We hypothesize that as a result of these initiatives there would be both an increase in the number of medical institutions, located in communities with ahigh percentage of the Hispanic population, and also an increase in the median household income of racial and ethnic minorities.

$\boldsymbol{H} 2 \boldsymbol{a}$ - Hispanics will have access to a greater number of medical institutions than they had before.

$\boldsymbol{H 3 a}$ - Hispanics will have a higher socioeconomic status than they had historically.

Researchers have used cumulative inequality to investigate how it affects the life trajectories of children $[26,29]$ and ethnic minorities [24]. The cumulative inequality is a function of the years of facing a disadvantage. After a set time the inequality is so large that it is very difficult to diminish it. There have been significant improvements in healthcare availability over the years. Horbar et al [42] documented an increase in performance of hospital care over a decade (20052014). However, the improvements were unequal among racial groups [43] with significant differences between Anglo and Hispanic/ African American minorities. Early advantages of medical institutions and higher income for the Anglo population [43, 44] have led to a cumulative disadvantage effect for Hispanics, such that even if they have higher income and more medical institutions it does not result in an increase in their healthcare status. Here we see the cumulative inequality play out clearly - Advantages for some have resulted in disadvantages for others. Based on this cumulative inequality, we hypothesize that even with increases in healthcare facilities and socioeconomic status, Hispanic population will still have increased instances of Covid-19 infections.

$\boldsymbol{H} 2 \boldsymbol{b}$-Access to a greater number of medical institutions will still have a positive relationship with the Coivd-19 infection rate among Hispanics

$\boldsymbol{H} 3 \boldsymbol{b}$ - Higher socioeconomic status will still have a positive relationship with the Coivd-19 infection rate among Hispanics.

We use a cumulative inequality-based weathering (CIW) framework to define our research model (see Figure 1) and guide our data analysis when we conduct our empirical case comparisons. Education is an important component of socioeconomic status [30], however income and education have been shown to be correlated [40] and hence for parsimony, we only used income in our empirical analysis. Our model considers the impact on ethnic minorities of the years consistent weathering in the form of lesser opportunities to access healthcare and how it has resulted in the formation of an institutional cumulative inequality for Hispanics that is so difficult to surmount in times of a pandemic.

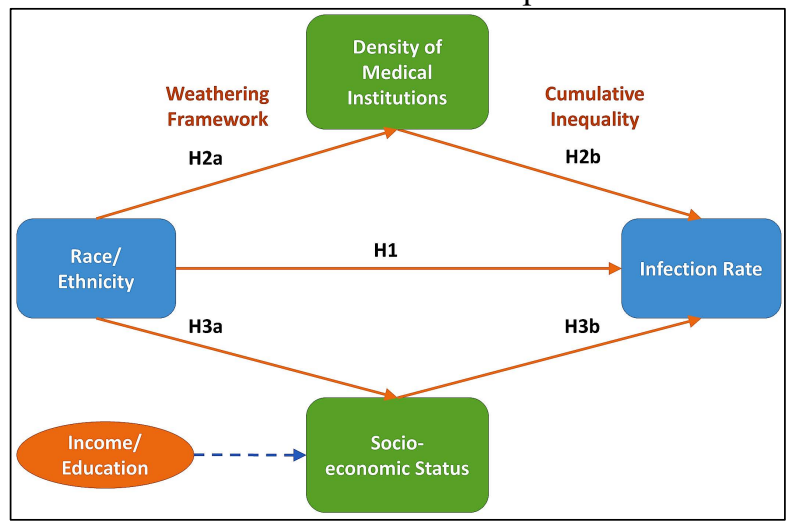

Figure 1. CIW Research Model

\section{Methodology}

We describe our data collection, analysis, and research methodology followed by us to undertake a qualitative case study [31] in the context of representative US population data which we refer to as empirical case comparisons (ECCs) in this paper.

\subsection{Data Collection}

We collected verified publicly available data published by US government agencies for all our indicator variables in our research model. Since the Covid-19 pandemic is still an ongoing health emergency the data on racial and ethnic minorities are currently segregated across various private and government domains [20, 32]. To have a clearer picture we standardized one source of data that is continually 
updated, verified, and published by The New York Times [33]. Table 1 presents our data outline.

Table 1. Data Outline

\begin{tabular}{|l|l|}
\hline Data & Agency \\
\hline $\begin{array}{l}\text { Median Household Income/ } \\
\text { Health Insurance Coverage }\end{array}$ & US Census \\
\hline $\begin{array}{l}\text { US Hospital General } \\
\text { Information }\end{array}$ & $\begin{array}{l}\text { County Information } \\
\text { Program }\end{array}$ \\
\hline $\begin{array}{l}\text { Demographic Racial/Ethnic } \\
\text { Population Parameters }\end{array}$ & US Census \\
\hline Covid-19 Infection Rate & $\begin{array}{l}\text { The New York } \\
\text { Times }\end{array}$ \\
\hline
\end{tabular}

\subsection{Empirical Case Comparisons}

For our case comparisons we use the following methodology: 1) From the US Census data we extracted demographic data (race, median income) for the Southern State and its various counties. 2) From this data we segregated counties according to the percentage of population ( $\%$ White only, not Hispanic or Latino; and $\%$ Hispanic or Latino). 3) Due to a diverse nature of the population in the Southern State with high racial diversity (Asian, African American, Hispanics and Whites), we set a population majority identifier of greater than $45 \%$. If Hispanics in a county made up more than $45 \%$ of the county's total population, we considered it a Hispanic majority county. We used the same criteria for determining White majority counties. 4) We then extracted the representative counties with similar population count such that the pair of White and Hispanic county had an equal population roughly in the ratio 1:1. This helped us to make an equivalent comparison within both the counties.

We compare the representative US individual and county population sample, based on US Census data, and show how over the years our indicator variables used in the research model have changed. We look at the rise in income levels of racial and ethnic minorities. We also look at a rise in the number of medical institutions located in areas where a higher percentage of the population is from racial and ethnic communities. We correlate this data with an increase in governmentprovided health insurance coverage and with the Covid19 data. The data from the Covid-19 dataset is used to draw up an infection rate which a marker for the number of people affected by the virus. Here affected means those who have contracted the virus and been tested as positive and also those who have succumbed to it. We have not considered education levels for these case comparisons, on account of its correlation with income [40], for parsimony.

From our access case comparisons in Table 2, we see that though the access of number of medical institutions and median household income increased for both Hispanic and Anglo populations there was no increase observed in the insurance coverage of Hispanics whereas, the Anglo population recorded an increase in the said indicator. This data is taken from a US Census report published by the United States Department of Commerce for the state of insurance coverage among the US population in 2017.

In Table 3, we see a representative case comparison of two US counties in a southern state. The counties were chosen according to the following guideline - one with a majority Hispanic population and the other with the majority Anglo population. The data again is from the latest current US Census projections for the year 2019. During the initial period of the Covid-19 pandemic there did not exist government database on coronavirus cases, and a team of New York Times journalists attempted to track every case [45]. The collated data was one of the first few sources of records that had minute details. With case counts segregated by the county level, which is the level of analysis in this study, the data has been since used in reputable research from National Bureau of Economic Research [46]. We use the data for the Covid-19 infections and deaths are taken from this New York Times dataset for the date July 14, 2020.This is representative of county-level data which corresponds to our representative case counties.

The Infection rate is calculated by using the following approach:

\begin{tabular}{|l|c|c|}
\hline Infection Rate $=$ & Infections + Deaths & X 100 \\
\cline { 2 - 2 } & Total Population & \\
\hline
\end{tabular}

As we see from the data, the infection rate for County A with a higher percentage of Hispanics is 1.06 as compared to County $\mathrm{B}$ with a higher percentage of the Anglo population, whose rate is 0.92 . This empirical analysis shows the existence of a cumulative inequalitybased weathering effect in the representative US Population.

\begin{tabular}{|l|c|c|c|}
\multicolumn{1}{c}{ Table 2. Access Case Comparisons } \\
\hline \multirow{1}{*}{} & $\begin{array}{c}\text { Medical } \\
\text { Institutions } \\
\text { Increased } \\
(\mathrm{Y} / \mathrm{N})\end{array}$ & $\begin{array}{c}\text { Median } \\
\text { Income } \\
\text { Increased } \\
(\mathrm{Y} / \mathrm{N})\end{array}$ & $\begin{array}{c}\text { Insurance } \\
\text { Coverage } \\
\text { Increased } \\
(\mathrm{Y} / \mathrm{N})\end{array}$ \\
\hline Hispanics & $\mathrm{Y}$ & $\mathrm{Y}$ & $\mathrm{N}$ \\
\hline Anglo & $\mathrm{Y}$ & $\mathrm{Y}$ & $\mathrm{Y}$ \\
\hline
\end{tabular}

\section{Discussion}

We used a qualitative case study methodology [31] to conduct empirical case comparisons using representative US Population sample markers and representative US County information. On analyzing the results from the case there were many surprising and counterintuitive findings. 
Cumulative Inequality causing Hispanic Adversity in Healthcare

Table 3. Representative Counties Comparison

\begin{tabular}{|c|c|c|c|c|c|c|}
\hline County & Hispanics & Anglo & Total Population & Infections & Deaths & Infection Rate (\%) \\
\hline A & 1216157 & 542963 & 2003554 & 21079 & 201 & 1.06 \\
\hline B & 620242 & 952439 & 2102515 & 19014 & 272 & 0.92 \\
\hline
\end{tabular}

\subsection{Findings}

On comparing the health and socioeconomic indicators across the racial and ethnic groups we found that even though the level of healthcare facilities and the median household income of both Anglo and Hispanic communities increased over the years but it did not lead to a subsequent increase in the level of governmentprovided insurance coverage for Hispanics. This shows support for our hypothesis $\mathrm{H} 2 \mathrm{a}$ and $\mathrm{H} 3 \mathrm{a}$ where Hispanic dominant counties have more medical institutions as well as have a higher household income than they had historically. When we compared representative county information, we also found similar results where county A with a majority Hispanic population had a higher infection rate $(1.06 \%)$ than county $\mathrm{B}(0.92 \%)$ which was predominantly made up of Anglo population. This comparison too showed support for our hypothesis $\mathrm{H} 1$, $\mathrm{H} 2 \mathrm{~b}$, and $\mathrm{H} 3 \mathrm{~b}$. We decided to base our data analysis on small and representative empirical case comparisons (ECCs) because we know that racial inequality is not a small topic and has considerable research literature in the epidemiology and social science domain. However, we use the insights from these ECCs as a starting point to open a new line of investigation focused on the importance of identifying institutional factors of disparities in the healthcare sector, and using technology as a way to bridge this gap by developing and enhancing current models of healthcare to ensure ubiquitous innovations and digital inclusion of all races. With respect to this we propose our new model (Digital inclusion and Access to Technology for Equitable healthcare delivery - DATE) for addressing disparities now and for the future in section 6.3.

From our ECCs analysis, we answer our research questions as follow: 1) Since a rise in the individual socioeconomic characteristic of median household income did not correspond to an increase in insurance coverage; we can say that even when individual attributes of ethnic minorities are better they still are not able to get access to a higher level of healthcare facilities. 2) The inability of individual indicators to explain why Hispanics are disproportionately affected by Covid-19 points to the existence of institutional factors that prevent ethnic communities from getting the same level of care as Anglo populations. 3) We see from our analysis that a rise in income did not correspond to either higher insurance coverage or a lesser infection rate. This shows that cumulative inequality has indeed taken hold in these communities because of them being subjected to years of the weathering effect. This has resultantly made the Hispanic adversity even greater in times of this pandemic.

\subsection{Contributions}

To the best of our knowledge, this work is the first to combine the two similar paradigms of the cumulative inequality theory [6] and the weathering hypothesis [7] to study racial inequality. We have formulated a CIW research model that seeks to investigate cumulative inequality that seeps in as a result of enduring a weathering effect for a long period of time. This long period can be considered as decades as the effect is shown to be generational in some studies [25]. From our ECCs we can state that the level of cumulative inequality will go on increasing if it is not checked at the very outset. The second contribution relates to our ECCs analysis pointing to the existence of an institutional factor that can be used to study racial and ethnic disparities and inequality in healthcare and other allied sectors. We saw that socioeconomic factors alone are not sufficient to predict the level of healthcare afforded to minorities in the country. Previous works have focused on studying these socio-economic factors from an individual perspective. However, there is a need to analyze racial inequality from an organizational and institutional level. This is because individual factors are dynamic and keep changing year on year but if institutional factors change it can cause serious harm to the community as evidence by hospitals that stopped operating in ethnic neighborhoods [21].

\subsection{Digital inclusion and $\underline{A}$ ccess to $\underline{T}$ echnology for Equitable healthcare (DATE)}

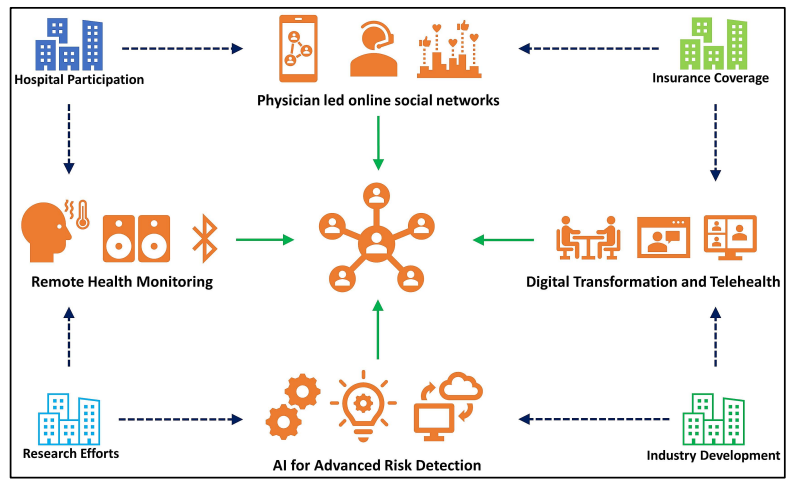

Figure 2. DATE Model for Ubiquitous Healthcare Innovation 


\section{Cumulative Inequality causing Hispanic Adversity in Healthcare}

Information Technology is a great leveler as it does not discriminate and anybody who learns how to use it can reap the benefits of its utilization. IT can be effectively used to bring people from different racial, ethnic, and economic backgrounds together. However, much like the disparity in the healthcare sector we see a disparity in access and use of technology among racial and ethnic minorities [48]. Also, availability of technology is an important factor determining its continued usage [49] which makes the technology disparity even more pronounced for the minorities. We propose the DATE model for increasing access to healthcare for minority populations. 1) Remote Health monitoring (Internet of Things - IoT): Smart medical devices like glucometers, heart rate, blood sugar and oxygen monitors, and a variety of smart wearable or ingestible devices are now readily available [50]. Hospitals should encourage the proliferation of such IoT devices for enhancing the last mile inclusion of disadvantaged and minorities within the healthcare sector. 2) Online Social Networks (Online Health Communities): People joining online health communities looking for information about their health diagnosis have increased exponentially [51]. Research in physician led online communities has shown that participation in such forums improves both patient wellbeing as well as patient-physician relationship [52]. Hospitals should participate and moderate such online communities to address the concerns of minorities who may not always have insurance coverage. 3) Digital Transformation and Telehealth: Covid-19 has catalyzed the rapid adoption of telehealth in the US healthcare industry [53]. However, insurance might not always cover these telehealth charges [54] and it is necessary to ensure that such non coverage does not exacerbate the existing racial disparities in the healthcare sector. 4) Artificial Intelligence and Advanced Risk Detection: The advent of big data and machine learning has revolutionized the healthcare domain. There have been studies that focus on predicting diseases [55] with sufficient accuracy. Government efforts should be enhanced to tap the potential of AI and medical data to model risk propagation of pathogenic outbreaks much like the novel coronavirus, so that advanced warnings can help lessen the impact of future pandemics. A study by Zhang \& Ram [56] utilized several data sources to learn asthma triggers and risk factors using machine learning techniques. Their results indicated that Hispanics are disproportionately affected by asthma. Thus, incorporating data from the medical domain is needed to learn such hidden identifiers that can tell us a great deal about at-risk populations and subsequently help to plan for the equitable access to healthcare. We believe the adoption of the DATE model at the Health Maintenance Organizations (HMO) levels will be an crucial first step to address not only the Hispanic adversity in the healthcare sector but also the lack of access for all minorities, racial or economic alike.

\subsection{Implications}

Our study provides support for the Theory of fundamental causes [34]. Phelan looks at racism as a factor for explaining inequalities in health. Our work also supports the idea of the existence of a superlative institutional factor that can be used to explain why certain groups are at a disadvantage as compared to others. Another implication of this work is in the mental health domain. Disparities exist in mental health counseling and care too, though they may not be as pronounced as in physiological care [8]. The level of care for mental health ailments is already low for the entire population but is striking among racial and ethnic minorities. Lack of proper care and counseling affects aspects of physiological care too. Depression, anxiety, and panic disorders have very real consequences for people which can hamper their normal life and routine. It is therefore imperative that research into this avenue is encouraged. Finally, 1) concrete steps should be taken by the government to improve healthcare access; 2) IT should be used for development/deployment of digitally inclusive technology to increase participation of minorities in the healthcare, which will ultimately help foster an era of reduced inequality. Racial data regarding access to healthcare, quality of service, rate of infection spread, areas hit the hardest, and systemic causes that force minorities into this adversity should be analyzed by the scientific and academic community [35].

\section{Limitations and Conclusion}

Since we collected data from the US Census there might be concerns of underreporting for the Hispanic population. In future work we can conduct cognitive surveys and interviews with minority populations and sample the information from them to reinforce our findings. Another limitation is that since Covid-19 is a developing situation there is a lack of race wise data from official sources and the reason for a lack of comparable counties. This can be addressed by continually updating our research methods and data sources in future studies. However, we believe the insights from our study are useful and can help to drive digital inclusion efforts aimed at addressing the inequality in the healthcare domain.

Individual socioeconomic characteristics such as Median Household Income are not sufficient to explain the disparate levels of care received by the racial and ethnic minority population groups in the country. A cumulative inequality is consistent for Hispanic 


\section{Cumulative Inequality causing Hispanic Adversity in Healthcare}

communities and does not depend on their socioeconomic levels. Also, the mere existence of healthcare facilities and services does not correspond to racial minorities using the said services. Further, we observed an increase in median household income for Hispanics, but it was not followed by a rise in government-provided healthcare coverage. Such counterintuitive insights lead us to conclude that there exist institutional factors that affect the level and quality of healthcare services available to the Hispanic community.

\section{References and Acknowledgement}

- The work of Y. Guo is partially supported by National Science Foundation under grant number CNS-2029685.

- The work of Y. Gong is partially supported by National Science Foundation under grant number CNS-1850523 and CNS-2029685.

[1] The Wall Street Journal (2020, July 13). As Covid-19 Cases Surge, Latino Communities Feel the Brunt. Retrieved from https://www.wsj.com/articles/as-covid-19-cases-surgelatino-communities-feel-the-brunt-11594632601. Accessed on 2020, July 14.

[2] Matthew, D. B. (2018). Just medicine: A cure for racial inequality in American health care. NYU Press.

[3] Nazroo, J. Y. (2003). The structuring of ethnic inequalities in health: economic position, racial discrimination, and racism. American journal of public health, 93(2), 277-284.

[4] Hayward, M. D., Miles, T. P., Crimmins, E. M., \& Yang, Y. (2000). The significance of socioeconomic status in explaining the racial gap in chronic health conditions. American sociological review, 910-930.

[5] Fiscella, K., Franks, P., Gold, M. R., \& Clancy, C. M. (2000). Inequality in quality: addressing socioeconomic, racial, and ethnic disparities in health care. Jama, 283(19), 2579-2584.

[6] Merton, R. K. (1988). The Matthew effect in science, II: Cumulative advantage and the symbolism of intellectual property. isis, 79(4), 606-623.

[7] Geronimus, A. T. (1992). The weathering hypothesis and the health of African-American women and infants: evidence and speculations. Ethnicity \& disease, 2(3), 207-221.

[8] Williams, D. R., Yu, Y., Jackson, J. S., \& Anderson, N. B. (1997). Racial differences in physical and mental health: Socio-economic status, stress and discrimination. Journal of health psychology, 2(3), 335-351.

[9] Phelan, J. C., \& Link, B. G. (2015). Is racism a fundamental cause of inequalities in health?. Annual Review of Sociology, 41, 311-330.

[10] Paradies, Y., Truong, M., \& Priest, N. (2014). A systematic review of the extent and measurement of healthcare provider racism. Journal of general internal medicine, 29(2), 364-387.

[11] Hayward, M. D., Miles, T. P., Crimmins, E. M., \& Yang, Y. (2000). The significance of socioeconomic status in explaining the racial gap in chronic health conditions. American sociological review, 910-930.
[12] Fiscella, K., Franks, P., Gold, M. R., \& Clancy, C. M. (2000). Inequality in quality: addressing socioeconomic, racial, and ethnic disparities in health care. Jama, 283(19), 2579-2584.

[13] Krieger, N. (2012). Methods for the scientific study of discrimination and health: an ecosocial approach. American journal of public health, 102(5), 936-944.

[14] Geronimus, A. T. (2001). Understanding and eliminating racial inequalities in women's health in the United States: the role of the weathering conceptual framework. Journal of the American Medical Women's Association (1972), 56(4), 133.

[15] Kail, B. L., \& Taylor, M. G. (2014). Cumulative inequality and racial disparities in health: Private insurance coverage and black/white differences in functional limitations. Journals of Gerontology Series B: Psychological Sciences and Social Sciences, 69(5), 798-808.

[16] Williams, D. R., \& Jackson, P. B. (2005). Social sources of racial disparities in health. Health affairs, 24(2), 325-334.

[17] Williams, D. R., \& Cooper, L. A. (2019). Reducing racial inequities in health: Using what we already know to take action. International journal of environmental research and public health, 16(4), 606.

[18] CNN (2020, May 18). Understanding why Latinos are so hard hit by Covid-19. Retrieved from https://www.cnn.com/2020/05/18/opinions/latinos-covid-19impact-morales/index.html. Accessed on 2020, July 12.

[19] Pew Research Center (2020, Mar 24). Hispanics more likely than Americans overall to see coronavirus as a major threat to health and finances. Retrieved from https://www.pewresearch.org/fact-

tank/2020/03/24/hispanics-more-likely-than-americans overall-to-see-coronavirus-as-a-major-threat-to-health-andfinances/. Accessed on 2020, July 12.

[20] CDC (2020, Jun 25) COVID-19 in Racial and Ethnic Minority Groups. Retrieved from https://www.cdc.gov/coronavirus/2019-ncov/need-extraprecautions/racial-ethnic-minorities.html. Accessed on 2020, July 12 .

[21] Kennedy, B. R., Mathis, C. C., \& Woods, A. K. (2007). African Americans and their distrust of the health care system: healthcare for diverse populations. Journal of cultural diversity, 14(2).

[22] Washington Post (2020, July 12). Latinos hit hard by coronavirus, but Chicago suggests it's even worse than it looks. Retrieved from https://www.washingtonpost.com/health/latinos-hit-hard-bycoronavirus-but-chicago-suggests-its-even-worse-than-itlooks/2020/07/10/a4ae1b62-bfa6-11 ea-b4f6cb39cd8940fb_story.html. Accessed on 2020, Jul 13.

[23] Merton, R. K. (1968). The Matthew effect in science: The reward and communication systems of science are considered. Science, 159(3810), 56-63.

[24] Kail, B. L., \& Taylor, M. G. (2014). Cumulative inequality and racial disparities in health: Private insurance coverage and black/white differences in functional limitations. Journals of Gerontology Series B: Psychological Sciences and Social Sciences, 69(5), 798-808.

[25] Gilligan, M., Karraker, A., \& Jasper, A. (2018). Linked lives and cumulative inequality: A multigenerational family life course framework. Journal of family theory \& review, $10(1), 111-125$. 


\section{Cumulative Inequality causing Hispanic Adversity in Healthcare}

[26] Jackson, M. I. (2015). Cumulative inequality in child health and academic achievement. Journal of Health and Social Behavior, 56(2), 262-280.

[27] Buescher, P. A., \& Mittal, M. (2006). Racial disparities in birth outcomes increase with maternal age: recent data from North Carolina. NC Med J, 67(1), 16-20.

[28] Governing.com (2015, Apr 14). Why Urban Hospitals Are Leaving Cities for Fancy Suburbs. Retrieved from https://www.governing.com/topics/health-human-

services/why-urban-hospitals-are-leaving-cities-for-fancysuburbs.html. Accessed on 2020, Jul 10.

[29] Schafer, M. H., Ferraro, K. F., \& Mustillo, S. A. (2011). Children of misfortune: Early adversity and cumulative inequality in perceived life trajectories. American Journal of Sociology, 116(4), 1053-1091.

[30] Smedley, B. D., \& Syme, S. L. (2000). Understanding and reducing socioeconomic and racial/ethnic disparities in health. In Promoting health: Intervention strategies from social and behavioral research. National Academies Press (US).

[31] Baxter, P., \& Jack, S. (2008). Qualitative case study methodology: Study design and implementation for novice researchers. The qualitative report, 13(4), 544-559.

[32] https://coronavirus.jhu.edu/data

[33] https://github.com/nytimes/covid-19-data

[34] Phelan, J. C., \& Link, B. G. (2015). Is racism a fundamental cause of inequalities in health?. Annual Review of Sociology, 41, 311-330.

[35] Chowkwanyun, M., \& Reed Jr, A. L. (2020). Racial health disparities and Covid-19-caution and context. New England Journal of Medicine.

[36] Strully, K. W. (2011). Health care segregation and race disparities in infectious disease: the case of nursing homes and seasonal influenza vaccinations. Journal of health and social behavior, 52(4), 510-526.

[37] Acevedo-Garcia, D. (2000). Residential segregation and the epidemiology of infectious diseases. Social science \& medicine, 51(8), 1143-1161.

[38] Nelson, A. (2002). Unequal treatment: confronting racial and ethnic disparities in health care. Journal of the National Medical Association, 94(8), 666.

[39] Moy, E., \& Freeman, W. (2014). Federal investments to eliminate racial/ethnic health-care disparities. Public Health Reports, 129(1_suppl2), 62-70.

[40] https://www.mbda.gov/who-we-are/overview

[41] Kimm, S. Y., Obarzanek, E., Barton, B. A., Aston, C. E., Similo, S. L., Morrison, J. A., ... \& McMahon, R. P. (1996). Race, socioeconomic status, and obesity in 9-to 10-year-old girls: the NHLBI Growth and Health Study. Annals of epidemiology, 6(4), 266-275.

[42] Horbar, J. D., Edwards, E. M., Greenberg, L. T., Morrow, K. A., Soll, R. F., Buus-Frank, M. E., \& Buzas, J. S. (2017). Variation in performance of neonatal intensive care units in the United States. JAMA pediatrics, 171(3), e164396e164396.

[43] Subramanian, S. V., \& Kawachi, I. (2004). Income inequality and health: what have we learned so far? Epidemiologic reviews, 26(1), 78-91.

[44] Pickett, K. E., \& Wilkinson, R. G. (2015). Income inequality and health: a causal review. Social science \& medicine, 128, 316-326.
[45] https://www.nytimes.com/article/coronavirus-countydata-us.html

[46] Kahn, L. B., Lange, F., \& Wiczer, D. G. (2020). Labor Demand in the time of COVID-19: Evidence from vacancy postings and UI claims (No. w27061). National Bureau of Economic Research.

[47] Brezina, T., \& Winder, K. (2003). Economic disadvantage, status generalization, and negative racial stereotyping by white Americans. Social Psychology Quarterly, 402-418.

[48] Jackson, L. A., Zhao, Y., Kolenic III, A., Fitzgerald, H. E., Harold, R., \& Von Eye, A. (2008). Race, gender, and information technology use: The new digital divide. CyberPsychology \& Behavior, 11(4), 437-442.

[49] Hsieh, J. P. A., Rai, A., \& Keil, M. (2008). Understanding digital inequality: Comparing continued use behavioral models of the socio-economically advantaged and disadvantaged. MIS quarterly, 97-126.

[50] Haghi, M., Thurow, K., \& Stoll, R. (2017). Wearable devices in medical internet of things: scientific research and commercially available devices. Healthcare informatics research, 23(1), 4-15.

[51] Huang, K. Y., Chengalur-Smith, I., \& Pinsonneault, A. (2019). Sharing is caring: social support provision and companionship activities in healthcare virtual support communities. MIS Quarterly, 43(2), 395-424.

[52] Liu, Q. B., Liu, X., \& Guo, X. (2020). The Effects of Participating in a Physician-Driven Online Health Community in Managing Chronic Disease: Evidence from Two Natural Experiments. MIS Quarterly, 44(1).

[53] Wosik, J., Fudim, M., Cameron, B., Gellad, Z. F., Cho, A., Phinney, D., ... \& Katz, J. N. (2020). Telehealth Transformation: COVID-19 and the rise of Virtual Care. Journal of the American Medical Informatics Association, 27(6), 957-962.

[54] Lin, J. C., Kavousi, Y., Sullivan, B., \& Stevens, C. (2020). Analysis of outpatient telemedicine reimbursement in an integrated healthcare system. Annals of vascular surgery, 65, 100-106.

[55] Chen, M., Hao, Y., Hwang, K., Wang, L., \& Wang, L. (2017). Disease prediction by machine learning over big data from healthcare communities. Ieee Access, 5, 8869-8879.

[56] Zhang, W., \& Ram, S. (2020). A Comprehensive Analysis of Triggers and Risk Factors for Asthma Based on Machine Learning and Large Heterogeneous Data Sources. MIS Quarterly, 44(1).[57] Weinick, R. M., Jacobs, E. A., Stone, L. C., Ortega, A. N., \& Burstin, H. (2004). Hispanic healthcare disparities: challenging the myth of a monolithic Hispanic population. Medical care, 313-320.

[58] Du, X. L., Fang, S., Coker, A. L., Sanderson, M., Aragaki, C., Cormier, J. N., ... \& Chan, W. (2006). Racial disparity and socioeconomic status in association with survival in older men with local/regional stage prostate carcinoma: findings from a large community-based cohort. Cancer: Interdisciplinary International Journal of the American Cancer Society, 106(6), 1276-1285.

[59] Mitrani, V. (2009). Reducing health disparities for Hispanics through the development of culturally tailored interventions. Hispanic health care international: the official journal of the National Association of Hispanic Nurses. 7(1),2 